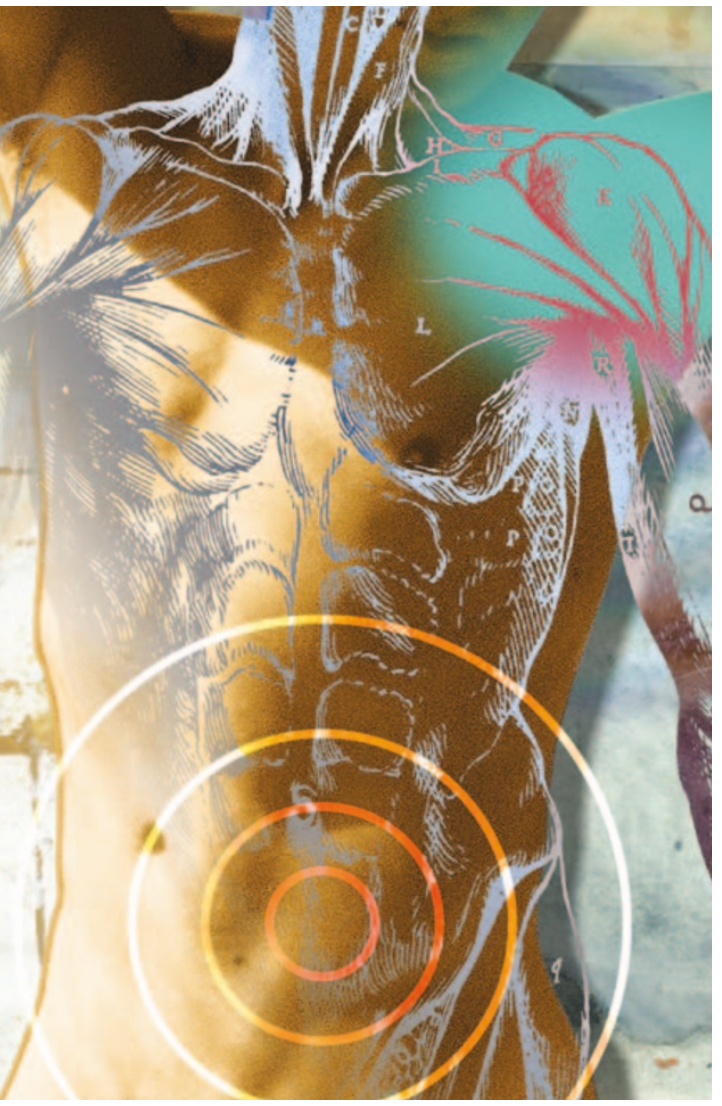

\title{
Local therapy for inflammatory bowel disease
}

Inflammatory bowel disease (IBD) refers to two chronic diseases: Crohn's disease (CD) and ulcerative colitis (UC). UC affects the large intestine, causing inflammation and ulceration of mucosal surfaces of the colon and rectum, whereas CD causes inflammation that extends into the deeper layers of the intestinal wall. Both diseases usually develop in early adult life. In a paper in the November issue of the Journal of Clinical Investigation, Fichtner-Feigl and colleagues report the successful treatment of both murine models of UC and CD with locally or systemically delivered nuclear factor- $\kappa \mathrm{B}$ (NF- $\mathrm{KB}$ ) decoy oligodeoxynucleotides (ODNs).

There is strong evidence that IBD is caused by inappropriate and excessive responses to antigens present in the normal bacterial flora. Despite the similarity in response to mucosal antigens, CD and UC have different pathophysiologies in terms of how $\mathrm{T}$ cells respond: $\mathrm{CD}$ is associated with a $\mathrm{T}_{\mathrm{H}} 1$ response, whereas
$\mathrm{UC}$ is associated with an atypical $\mathrm{T}_{\mathrm{H}} 2$ response. However, in both human IBD and in murine models of IBD, the inflammation is likely to depend on nuclear translocation and transcriptional activation of NF- $\kappa \mathrm{B}$ and related proteins. The authors therefore pursued a strategy of blocking NF- $\kappa \mathrm{B}$ activity for both major forms of IBD.

The authors explored the role of NF- $\mathrm{\kappa B}$ activity in $\mathrm{CD}$ and UC by testing the capacity of NF- $\mathrm{KB}$ decoy ODNs to treat various murine models of IBD. They showed that intrarectal or intraperitoneal administration of decoy ODNs, which were encapsulated in a viral envelope, prevented and treated a model of acute and chronic trinitrobenzene sulphonic acid (TNBS)-induced colitis, as assessed by clinical course and effect on $T_{H} 1$ cytokine production. Even when given late in the course of a chronic model of UC, the decoy ODNs prevent the development of characteristic fibrosis. The authors also showed

\section{REGENERATION}

\section{A new stop signal}

Failure of axonal regeneration in the adult mammalian central nervous system (CNS) is partly due to the inhibitory effect of molecules associated with CNS myelin and the glial scar, but the underlying intracellular signalling mechanisms are not clear. Writing in Science, Koprivica and colleagues show that inhibition of regeneration is mediated by activation of the epidermal growth factor receptor (EGFR), and that EGFR inhibitors promote significant repair of injured optic nerve fibres.

The potent inhibitory effect of myelin and the glial scar on axonal growth can be easily studied in culture. When neurons are grown in the presence of either myelin or the active component of the glia scar, chondroitin sulphate proteoglycans (CSPGs), they will have little neurite arborization compared with their counterparts grown in control conditions. This easy assay has allowed Koprivica et al. to screen $\sim 400$ well-characterized small molecules for their capacity to reverse the inhibitory activity of myelin and CSPGs.

Surprisingly, several EGFR kinase inhibitors, including AG1478, PD168393 and erlotinib, enhanced neurite extension from dissociated rat cerebellar granule and dorsal root ganglion (DRG) neurons grown in the presence of either myelin or CSPGs. Moreoever, axonal growth of DRG neurons expressing a dominant-negative mutant form of EGFR was no longer blocked by myelin or CSPGs, indicating that EGFR kinase activity might be involved in the inhibition of regeneration.

This prompted the researchers to test the effect of myelin on EGFR phosphorylation in serum-starved cerebellar granule neurons. Both Nogo66 and oligodendrocyte myelin glycoprotein - myelin-associated inhibitory proteins - triggered rapid EGFR phosphorylation. This effect was not seen in neurons overexpressing a dominant-negative mutant form of $\mathrm{NgR}$, the common ligandbinding component of the receptor complexes, which indicates that $\mathrm{NgR}$ or its functional homologues might be necessary for EGFR activation.
Do EGFR inhibitors have an effect on nerve repair in vivo? To test this, Koprivica et al. crushed the optic nerve in adult mice and applied gelform containing PD168393 at the lesion site immediately after injury. Two weeks later, there was substantial axonal growth in animals treated with the EGFR inhibitor, resulting in a ninefold increase in the number of regenerating fibres $0.25 \mu \mathrm{m}$ beyond the injury site compared with the control.

This surprising finding has important therapeutic implications for patients with spinal or other CNS injuries. As one of the EGFR inhibitors, erlotinib, has been approved by the US FDA for the treatment of lung cancer, its efficacy in nerve repair could soon be tested in clinical trials.

Jane Qiu, Associate Editor Nature Reviews Neuroscience

\section{(7) References and links} ORIGINAL RESEARCH PAPER Koprivica, V. et al. EGFR activation mediates inhibition of axon regeneration by myelin and chondroitin sulfate proteoglycans. Science $\mathbf{3 1 0}$, 106-110 (2005)

FURTHER READING Filbin, M. T. Myelin-associated inhibitors of axonal regeneration in the adult mammalian CNS. Nature Rev. Neurosci. 4, 703-713 (2003) WEB SITE He's laboratory:

http://www.hms.harvard.edu/dms/neuroscience/fac_he.htm 
that the decoy ODNs prevented and treated oxazolone-induced colitis, which is a $\mathrm{T}_{\mathrm{H}} 2$-mediated inflammatory process. In each case, decoy ODN administration led to inflammation-clearing effects, suggesting a therapeutic potency applicable to human IBD.

Importantly, treatment of TNBSinduced inflammation by intrarectal administration of NF- $\kappa \mathrm{B}$ decoy ODNs did not inhibit NF- $\kappa B$ in other organs. One of the effects of blocking $\mathrm{NF}-\kappa \mathrm{B}$ is the decrease of interleukin12 , a cytokine that has important anti-apoptotic effects of $\mathrm{T}_{\mathrm{H}} 1 \mathrm{~T}$ cells. Local delivery of the decoy ODNs still resulted in T-cell apoptosis, suggesting that such treatment is likely to have a durable therapeutic effect.

Melanie Brazil

(2) References and links ORIGINAL RESEARCH PAPER Fichtner-Feigl, S. et al. Treatment of murine Th1- and Th2-mediated inflammatory bowel disease with NF-KB decoy oligonucleotides. J. Clin. Invest. 115, 3057-3071 (2005)

FURTHER READING Bouma, G. \& Strober, W. The immunological and genetic basis of inflammatory bowel disease. Nature Rev. Immunol. 3, 521-533 (2003)

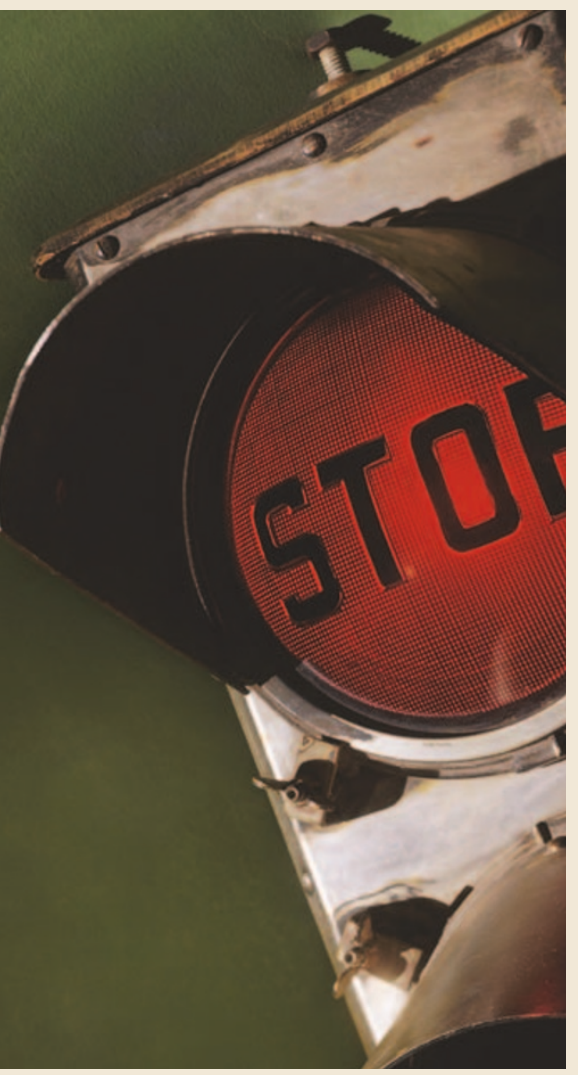

\section{COMPUTATIONAL CHEMISTRY}

\section{Ready, steady, screen!}

Computational screening methods are established as a complementary approach to high-throughput screening for identifying potential active compounds, but rarely have the two strategies been directly compared. Investigating this issue was one aim of a recent unique competition, in which entrants were challenged to computationally predict the results of a high-throughput screen, and selected results are presented in a special issue of the Journal of Biomolecular Screening.

The competition was based on data from high-throughput screens of two 50,000compound libraries against dihydrofolate reductase (DHFR), an established drug target for the treatment of bacterial infections, cancer and malaria. Entrants were given the structures and measured levels of inhibition for the compounds in a 'training' set of 50,000 diverse compounds that contained 12 competitive DHFR inhibitors to aid in validating computational screening strategies. The structures of the second set of 50,000 compounds were also provided, but not the screening results, and the test was to predict these results.

The approaches used by the competition entrants, the most successful of which are featured in the issue, fell broadly into several categories: those based on quantitative structure-activity relationship analysis; on molecular similarity; on docking; and combinations of these approaches. However, overall, no group predicted more than $15 \%$ of the apparent inhibitors in the test set.

Despite this apparent low rate of success, several valuable insights were obtained, including possible reasons for the outcome. Bender et al., who used a fragment-based similarity searching method, found that a key issue was a lack of similarity in the distribution of chemical features of the training and test sets. And Brenk and colleagues, whose strategy was based on docking, predicted that few true inhibitors would be found in the test screen, which indeed turned out to be the case, as none of the hits identified in this screen were validated as potent competitive inhibitors. Furthermore, this group predicted that some of the hits that were identified in the experimental screen could be false positives owing to compound aggregation, highlighting the need for procedures that can cull such molecules from screening libraries.
In addition to such insights, key areas for the improvement of similar competitions were suggested by the judges. Most importantly, prescreening all the compounds and then creating training and test sets based on equal distributions of experimental data and chemical properties, and ensuring the presence of validated competitive inhibitors in both, would allow better comparison of the predictive ability of different strategies. Using additional related and unrelated targets would also be valuable in reducing potential biases owing to the nature of the binding site and in allowing the ability of different approaches to discriminate between selective inhibitors to be evaluated.

Peter Kirkpatrick

\section{D) References and links}

ORIGINAL RESEARCh PAPERS Elowe, N. H. et al. Experimental screening of dihydrofolate reductase yields a "test set" of 50,000 small molecules for a computational data-mining and docking competition. J. Biomol. Screen. 10, 653-657 (2005) | Bender, A. et al. Screening for dihydrofolate reductase inhibitors using MOLPRINT 2D, a fast fragment-based method employing the naiive Bayesian classifier: limitations of the descriptor and the importance of balanced chemistry in training and test sets. J. Biomol. Screen. 10, 658-666 (2005) | Brenk, R. et al. Here be dragons: docking and screening in an uncharted region of chemical space. J. Biomol. Screen. 10, 667-674 (2005) | Bernacki, K. et al. Virtual ligand screening against Escherichia coli dihydrofolate reductase: improving docking enrichment using physics-based methods. J. Biomol. Screen. 10, 675-681 (2005) | Rogers, D. et al. Using extended-connectivity fingerprints with Laplacian-modified Bayesian analysis in high-throughput screening follow-up. J. Biomol. Screen. 10, 682-686 (2005)

FURTHER READING Parker, C. N. McMaster university data-mining and docking competition: computational models on the catwalk. J. Biomol. Screen. 10, 647-648 (2005) | Lang, P. T. et al. Evaluating the high-throughput screening computations. J. Biomol. Screen. 10 649-652 (2005)

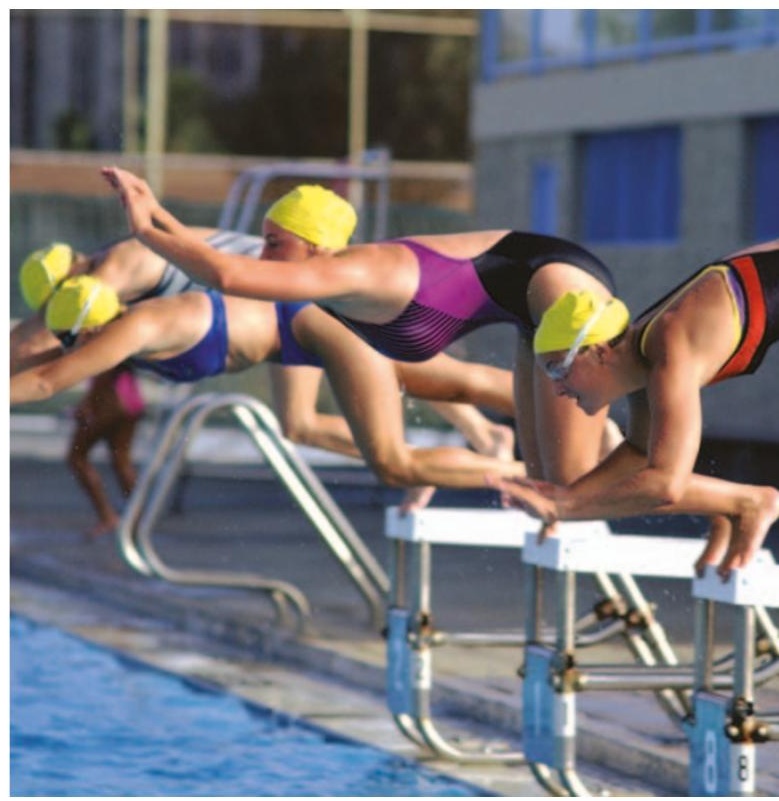

